\title{
Photonics: From European support to industrial technology leadership
}

\author{
Photonics support in the European programmes dates back to the early $90^{\mathrm{s}}$, \\ though the "Telematics" and "Esprit" initiatives (1983 to 1998) primarily \\ focused on the then emerging field of optical telecommunication, \\ fibre optics, optoelectronic, detectors and III-Vs and semiconductor lasers.
}

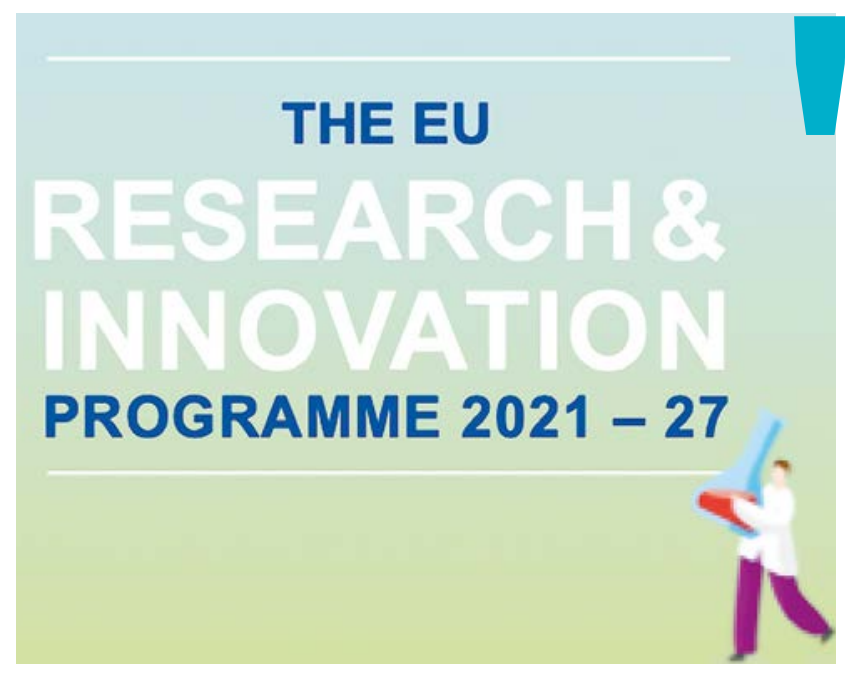

Henri RAJBENBACH, John MAGAN and Werner STEINHOEGL .

European Commission,

Directorate General CONNECT (Communications

Networks, Content \& Technology),

Unit A3 - Microelectronics and Photonics Industry 


\section{A SOLUTION FOR EACH METROLOGY PROJECT}

Nowadays, the capability to accurately measure optical systems and components is crucial at each development stage: sample qualification, prototype optimization and final integration. Therefore, the test stations need to fit the metrology requirements both in terms of technical specifications, and practical implementation. Technically, the stations should be adapted in terms of wavelength, sampling, accuracy... In practice, some people need a complete, ready-to-use, and turnkey solution, while others are looking for something more flexible.

Leveraging more than 15 years of experience in the development of wavefront metrology solutions, Phasics is answering this challenge with its broad spectrum of measurement tools: from standalone wavefront sensors to fully integrated test stations, including a new versatile intermediate solution.

- The SID4 wavefront sensors range, available from UV to

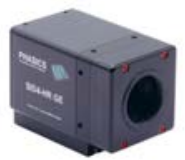
LWIR, with various samplings. These high resolution achromatic wavefront sensors can easily be integrated in any set-up.

- The Kaleo Kit, a brand-new modular and compact solution. Choosing between a large variety of modules

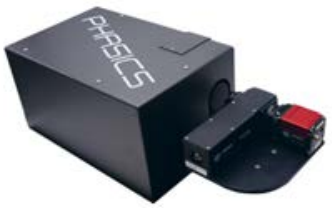

(SID4 wavefront sensors, $\mathrm{R}$-cube illumination modules, beam expander, and focusing modules) the user assembles his own test station according to his requirements.

- The Kaleo MultiWave, a complete ready-to-use, dynamic

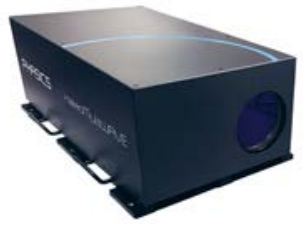
interferometer integrating up to 8 test wavelengths on a unique test station. It measures TWE and RWE at the sample design wavelength.

Thanks to all the available options in terms of specifications and implementation, Phasics solutions benefit to a large variety of industries: smartphones, $\mathrm{AR} / \mathrm{VR}$, aerospace, autonomous driving, astronomy to name a few.

\section{CONTACT}

PHASICS - Phone: +33 (0) 180750633

contact@phasics.fr -www.phasics.com
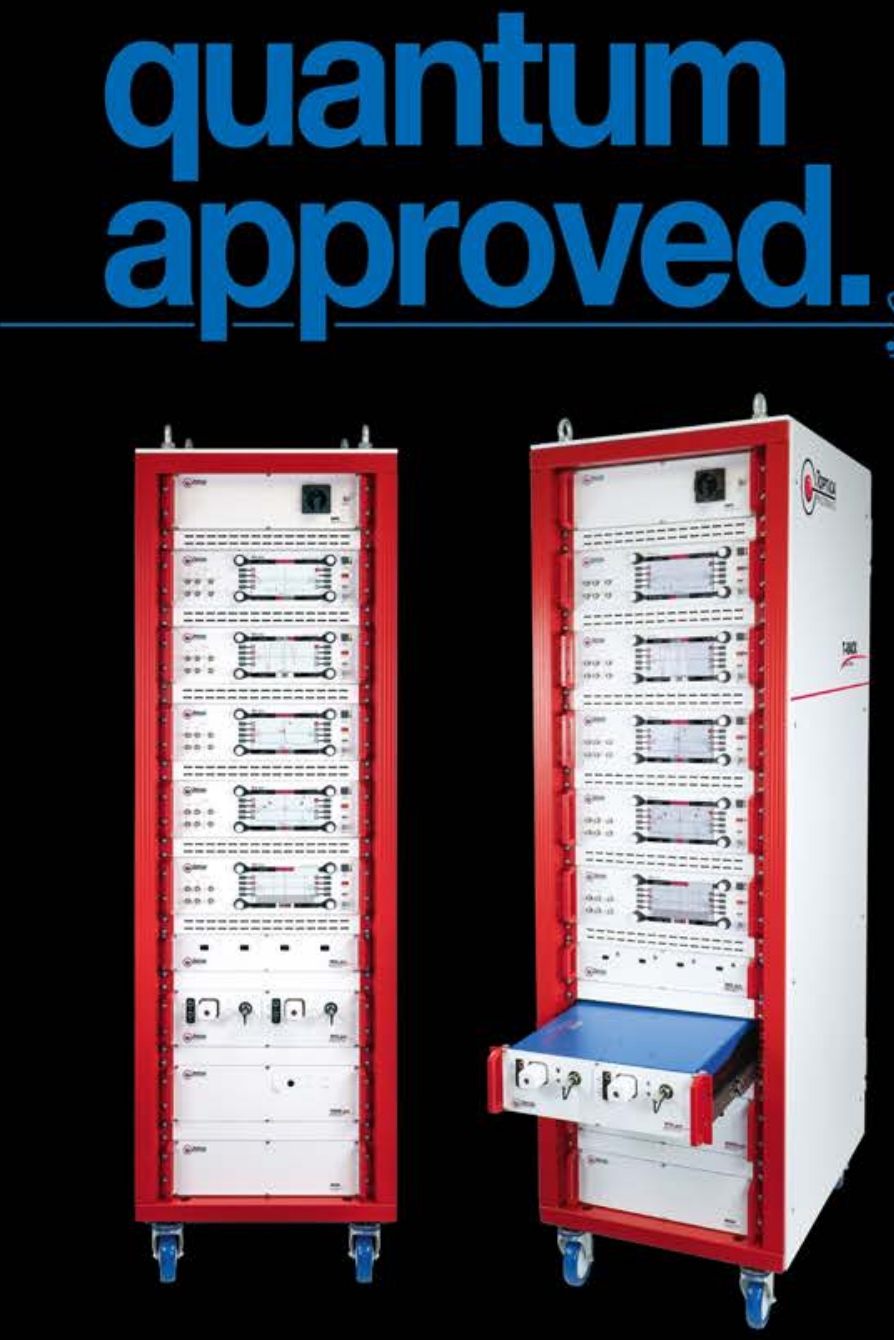

\section{Laser Rack Systems}

Quantum Technology meets Industry Standards

Our lasers do not need an optical table!

The T-RACK is the perfect home for TOPTICA's high-end tunable diode lasers and frequency combs in a modular 19" form factor. Pick yours!

- Tunable Diode Laser Systems

- Frequency Comb Systems

- Wavelength Meters

- Locking Electronics

- 330 .. $1770 \mathrm{~nm}$

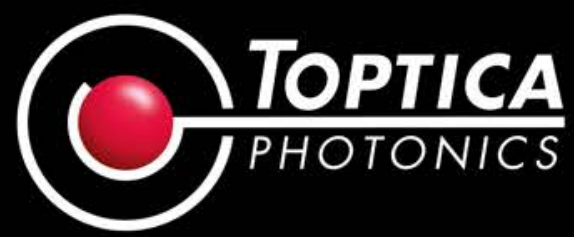


Now comes the future. What is next? What will happen to Photonics between 2021 and 2027?

Electrons and Photons need to work even closer together in ever-miniaturised systems. The Horizon Europe programme is the next horizon, for the period 2021-2027. A few topics will already be called for in the coming two years:In a topic centred on "Optical communication components" (26 m€), Innovation actions will develop ultra-dynamic photonic components and subsystems for data communication, using for example new optical wavelength bands, space division multiplexing, new integration schemes, optical switching and new switching paradigms, as solutions for time-deterministic and time-sensitive networks.

Advancing integration technologies will be the subject of the "PIC topic" (38 m€). Projects will address the co-integration of photonic and electronic, facilitating new applications in biomedical, environmental and industrial fields, making devices more power-efficient and bringing ground-breaking technologies within reach of entrepreneurial SMEs. Finally, at the crossroad of Photonics and Nanoelectronics, the "Advanced multi-sensing systems" topic (47 m€) will be calling for breakthroughs in sensor

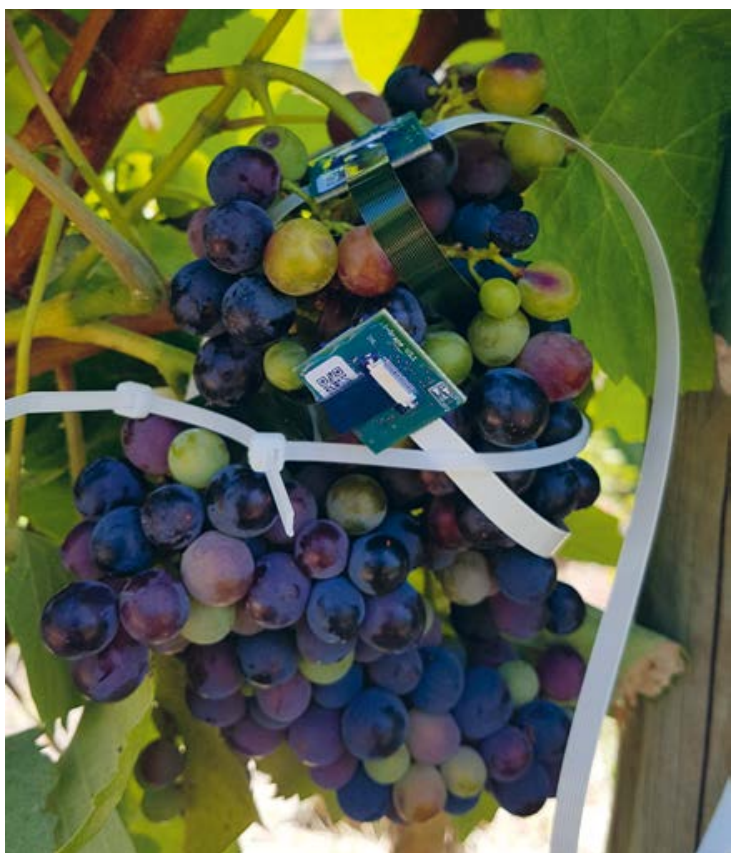

The ICT i-Grape project develops fully integrated and connected miniaturised low-cost, standalone microspectroscopic smart systems for grape maturation and vine hydric stress monitoring. The detection head integrates UV-VIS-NIR LED sources and photodiode/interference filter arrays at wafer level. It is connected with a loT processing unit for real-time online processing and monitoring. http://i-grape.eu

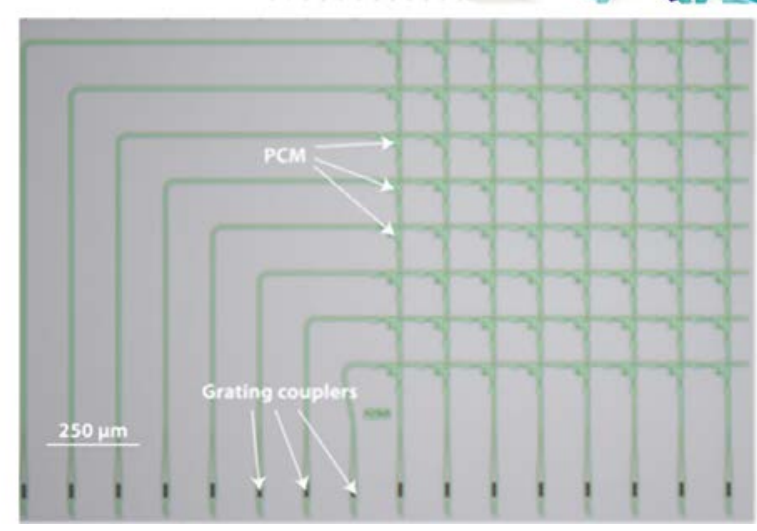

The ICT Fun-COMP project develops an all-photonic MatrixVector Multiplier (MVM) for neuromorphic, brain-like neural networks. Top shows the system schematic: a micro-comb laser source generates multiple wavelengths modulated according to the vector values and launched into the rows of the MVM array. Bottom shows the fabricated MVM array. Matrix elements are stored in the programmable nonvolatile states of phase-change material (PCM) cells; the MVM output emerges at the bottom of the columns. https://fun-comp.org/

systems by combining component development, system integration, packaging and cost-effective manufacturing processes. They would propose innovative approaches capable of acquiring, processing and interpreting vast amounts or fast changing sensory input data, while reducing significantly overall energy consumption. The sensing functionality builds on technologies related to light and will include integration with microelectronics, micro-nano-mechanical, micro-fluidic, magnetic, radio frequency or bio-chemical technologies.

To take Europe's photonics industry to 2030 and beyond, it will not be sufficient to focus solely on individual research projects. While the EU support for these projects has been of outstanding value and will continue, future focus will be even more closely on creating the ecosystems and markets of tomorrow. That means enabling the transfer of breakthroughs in photonics technology into successful products and services that create new jobs, resolving our most important societal challenges and improving the lives of European citizens. The new Photonic Partnership aims to speed up photonic innovations for a digital, green and healthy future in Europe, securing Europe's technological sovereignty, raising the competitiveness of Europe's economy and ensuring long-term job and prosperity creation. The past has been bright, the future is even brighter! 